\title{
Drawing Graphs with Vertices at Specified Positions and Crossings at Large Angles
}

\author{
Martin Fink ${ }^{1}$, Jan-Henrik Haunert ${ }^{1}$, Tamara Mchedlidze $^{2}$, \\ Joachim Spoerhase ${ }^{1}$, and Alexander Wolff ${ }^{1}$ \\ 1 Lehrstuhl für Informatik I, Universität Würzburg, Germany \\ ${ }^{2}$ Department of Mathematics, National Technical University of Athens, Greece
}

\section{Introduction}

In point-set-embeddability (PSE) problems one is given not just a graph that is to be drawn, but also a set of points in the plane that specify where the vertices of the graph can be placed. The problem class was introduced by Gritzmann et al. 3] twenty years ago. In their work and most other works on PSE problems, however, planarity of the output drawing was an essential requirement. Recent experiments on the readability of drawings [4] showed that polyline drawings with angles at edge crossings close to $90^{\circ}$ and a small number of bends per edge are just as readable as planar drawings. Motivated by these findings, Didimo et al. 2] recently introduced $R A C$ drawings where pairs of crossing edges must form a right angle and, more generally, $\alpha A C$ drawings (for $\left.\alpha \in\left(0,90^{\circ}\right]\right)$ where the crossing angle must be at least $\alpha$. As usual, edges may not overlap and may not go through vertices. We investigate the intersection of PSE and RAC $/ \alpha \mathrm{AC}$.

Specifically, we consider the problems $R A C P S E$ and $\alpha A C P S E$ defined as follows. Given an $n$-vertex graph $G=(V, E)$ and a set $S$ of $n$ points in the plane, determine whether there exists a bijection $\mu$ between $V$ and $S$, and a polyline drawing of $G$ so that each vertex $v$ is mapped to $\mu(v)$ and the drawing is RAC (or $\alpha \mathrm{AC}$ ). If such a drawing exists and the largest number of bends per edge in the drawing is $b$, we say that $G$ admits a $\mathrm{RAC}_{b}$ (or an $\alpha \mathrm{AC}_{b}$ ) embedding on $S$. If we insist on straight-line edges, the drawing is completely determined by a bijection between vertex and point set. If we allow bends, however, PSE is also interesting with mapping, that is, if we are given a bijection $\mu$ between vertex and point set. In order to measure the size of our drawings, we assume that the given set of $n$ points lies on a grid $\Gamma$ of size $n \times n$ and, in the output drawing, bends lie on a (potentially larger or finer) grid containing $\Gamma$. We further assume that no two points lie on the same horizontal or vertical line. We call such a point set an $n \times n$ grid point set.

\section{Results}

We can $\mathrm{RAC}_{3}$ embed any graph with $n$ vertices and $m$ edges on any $n \times n$ grid point set using any mapping and area $O\left((n+m)^{2}\right)$, see Fig. 1. Here, the idea is to have crossings only between segments with slopes +1 and -1 . By choosing the horizontal positions of the first and last bends of each edge (black boxes in 


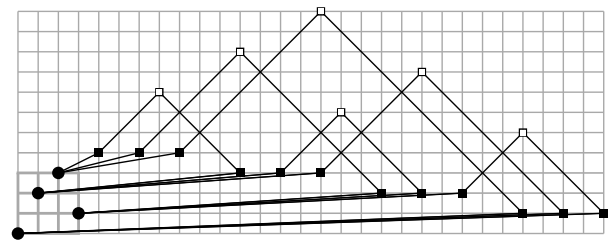

Fig. 1. $\mathrm{RAC}_{3}$ embedding of $K_{4}$

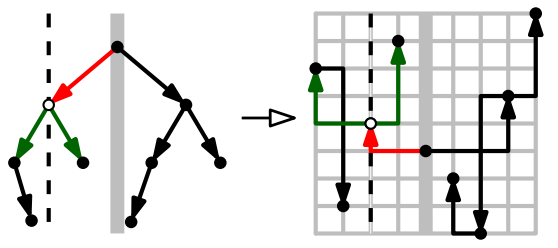

Fig. 2. Restricted $\mathrm{RAC}_{1}$ embedding

Fig. (1) so that they all have even Manhattan distance, we ensure that the middle bends (squares in Fig. 1) lie on grid points, and, thus, the drawing is valid.

In order to get 1-bend drawings (again for any mapping $\mu$ ), we refine the grid by a factor of $\lambda \in O(\cot \varepsilon)$, for a small angle $\varepsilon$, that is, we insert, at equal distances, $\lambda-1$ new rows and columns between each pair of consecutive old grid rows and columns, respectively. For each edge $u v$ of the given graph $G$, consider the point $p_{u v}$ in the same row as $\mu(u)$ and the same column as $\mu(v)$. Clearly, $p_{u v}$ lies on the original grid. We place the bend of $u v$ at one of the four new grid points that are diagonally adjacent to $p_{u v}$. This yields a $(\pi / 2-\varepsilon) \mathrm{AC}_{1}$ embedding of $G$ on the given grid point set $S$; the refined grid has size $O\left((\lambda n)^{2}\right)$.

We now turn to a restricted version of our problem where additionally every edge has to be drawn on grid lines, see Fig. 2 (right).

We show that every $n$-vertex binary tree admits a restricted $\mathrm{RAC}_{1}$ embedding on any $n \times n$ grid point set (which is not known for the planar case). This was independently shown by Di Giacomo et al. [1]. We simply view the given tree as a search tree for the points, sorted by x-coordinate, and draw the edges (directed away from the root) such that we enter each vertex vertically and leave it horizontally to the left and to the right to its at most two children, see Fig. 2,

By an old result of Vizing (1964), every graph of maximum degree 3 can be 4-edge-colored. We exploit this to construct restricted $\mathrm{RAC}_{2}$ embeddings of such graphs on any $n \times n$ grid point set even if the mapping is prescribed. We interpret each color as a direction (up, down, left, right) and, for each edge, we draw its first and third segment into this direction and the middle segment far enough.

\section{References}

1. Di Giacomo, E., Frati, F., Fulek, R., Grilli, L., Krug, M.: Orthogeodesic point-set embedding of trees. In: Speckmann, B., van Kreveld, M. (eds.) GD 2011. LNCS, vol. 7034, pp. 52-63. Springer, Heidelberg (2011)

2. Didimo, W., Eades, P., Liotta, G.: Drawing Graphs with Right Angle Crossings. In: Dehne, F., Gavrilova, M., Sack, J.-R., Tóth, C.D. (eds.) WADS 2009. LNCS, vol. 5664, pp. 206-217. Springer, Heidelberg (2009)

3. Gritzmann, P., Mohar, B., Pach, J., Pollack, R.: Embedding a planar triangulation with vertices at specified positions. Amer. Math. Mon. 98, 165-166 (1991)

4. Huang, W., Hong, S.H., Eades, P.: Effects of crossing angles. In: Proc. 7th Int. IEEE Asia-Pacific Symp. Inform. Visual (APVIS), pp. 41-46 (2008) 